\title{
Lecture Notes
}

in Control and Information Sciences

Editor: M. Thoma 
Springer-Verlag

London Ltd. 
Qing-Guo Wang, Tong Heng Lee and Kok Kiong Tan

Finite Spectrum Assignment for Time-Delay Systems 


\section{Series Advisory Board}
A. Bensoussan - M.J. Grimble - P. Kokotovic - H. Kwakernaak
J.L. Massey - Y.Z. Tsypkin

\section{Authors}

\section{Qing-Guo Wang \\ Tong Heng Lee}

Kok Kiong Tan

Department of Electrical Engineering, The National University of Singapore,
10 Kent Ridge Crescent, S (119260) Singapore

\section{ISBN 978-1-85233-065-1}

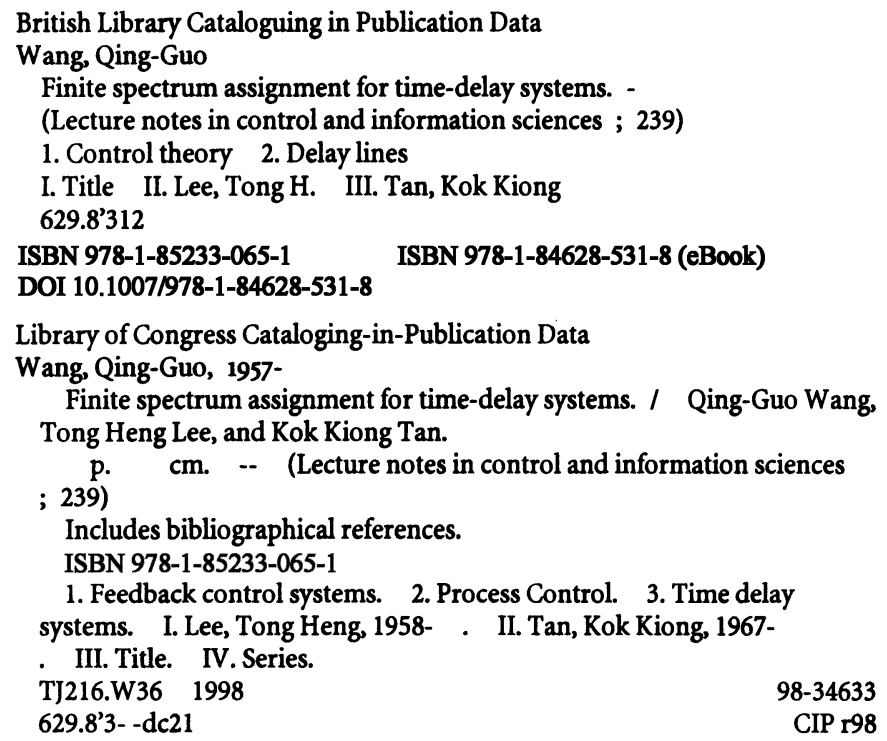

Apart from any fair dealing for the purposes of research or private study, or criticism or review, as permitted under the Copyright, Designs and Patents Act 1988, this publication may only be reproduced, stored or transmitted, in any form or by any means, with the prior permission in writing of the publishers, or in the case of reprographic reproduction in accordance with the terms of licences issued by the Copyright Licensing Agency. Enquiries concerning reproduction outside those terms should be sent to the publishers.

\section{c) Springer-Verlag London 1999 \\ Originally published by Springer-Verlag London Berlin Heidelberg in 1999}

The use of registered names, trademarks, etc. in this publication does not imply, even in the absence of a specific statement, that such names are exempt from the relevant laws and regulations and therefore free for general use.

The publisher makes no representation, express or implied, with regard to the accuracy of the information contained in this book and cannot accept any legal responsibility or liability for any errors or omissions that may be made.

Typesetting: Camera ready by authors 


\section{Preface}

The presence of considerable time delays in many industrial processes is well recognized and achievable performances of conventional unity feedback control systems are degraded if a process has a relatively large time delay compared to its time constants. In this case, dead time compensation is necessary in order to enhance the performances. The most popular scheme for such compensation is the Smith Predictor, but it is unsuitable for unstable or lightly damped processes because the compensated closed-loop system always contains the process poles themselves. An alternative scheme for delay elimination from the closed-loop is the finite spectrum assignment (FSA) strategy and it can arbitrarily assign the closed-loop spectrum. One may note that the SmithPredictor Control can be found in delay systems control books and many process control books, but the FSA control is rarely included in these books. It is therefore timely and desirable to fill this gap by writing a book which gives a comprehensive treatment of the FSA approach. This is useful and worthwhile since the FSA provides not only an alternative way but also certain advantages over the Smith-Predictor.

The book presents the state-of-the-art of the finite spectrum assignment for time-delay systems in frequency domain. It mainly contains those works carried out recently by the authors in this field. Most of them have been published and others are awaiting publication. They are assembled together and reorganized in such a way that the presentation is logical, smooth and systematic. The book covers the whole range of the topic from algorithms development to practical issues relating to the application of FSA such as auto-tuning and performance robustness. Both simulation and real-time implementation results are included for illustration of the FSA methodology. The pre-requisites are minimal and only knowledge of a first undergraduate control course is assumed. The book is thus readily accessible and practically useful for senior undergraduate and graduate students and engineers.

In what follows, the contents of the book will be briefly reviewed. Chapter 1 provides a short introduction to delay systems, the control strategies for these systems and practical issues expected in implementation. The FSA is one such control strategy for these systems and it first originated from Manitius and Olbrot (1979) in the time domain. The frequency domain version was proposed by Ichikawa (1985), but it can only be applied to single variable systems with distinct poles. A subsequent version of the algorithm extends the application to systems with multiple poles. Multivariable FSA is also desirable since many industrial systems are of multivariable nature. In addition, modification of the 
algorithm is also necessary to achieve asymptotic regulation and tracking as an ordinary FSA system usually has non-zero steady-state error in response to a step set-point change or load disturbance. Chapter 2 of the book is mainly a collection of the basic and extended versions of the FSA algorithms.

Automatic tuning and adaptation of PID controllers have been successfully applied to industrial processes. This adaptive technique is also needed for model-based advanced controllers such as the FSA if the same success is expected for their applications in industry, as time-varying time delays and/or dynamics often occur because of varying flows and/or operating conditions and require frequent re-tuning of the controllers. Chapters 3 and 4 deal with these practical issues expected in the implementation of FSA systems. Chapter 3 is concerned with non-parametric estimation of the process and fitting of transfer function models to the frequency response. Two useful and practical methods are described. The first method applies the basic idea due to Astrom (1982). The basic and now renowned relay feedback for PID auto-tuning is extended to identify one or more general points on the process Nyquist curve in the more interesting range of frequency, in similar efficiency as the basic relay method estimates the process critical point. The second method examined in the chapter is an online frequency response estimation method based on the use of Discrete Fourier Transform applied to transient signals in relay or step tests. Chapter 4 is focused on controller design, auto-tuning and adaptation based on the transfer functions fitted to the frequency response estimation by the methods described in Chapter 3. Applications of the techniques in process control are demonstrated by simulation and real-time implementations in these chapters.

In practical situations, models of industrial processes are never perfect and robustness of FSA systems in the presence of inevitable uncertainty should be addressed. The practical stability of finite spectrum-assigned delay systems is first considered in Chapter 5. It is shown that such systems suffer from a practical stability problem, i.e. an infinitesimal perturbation in the process may destabilize a nominally stable system. The conditions for practical stability are related to structural aspects of the process and model. Similarly, the conditions for robust stability and performance robustness for perturbations of arbitrary magnitude are derived. These provide design guidelines in the development of the control system.

This book would not be possible without the help of certain people to whom the authors would like to express their appreciation. In particular, we would like to thank Mr. Bi Qiang and Mr. Zou Biao for their works related to Chapters 3 and 4 . The editing process would not have been as smooth without the generous assistance of Mr. Zhang Li, Mr. C. H. Gan and Mr. Zhang Yong. 


\section{Contents}

1 Time-Delay Systems 1

1.1 Introduction . . . . . . . . . . . . . . 1

1.2 Control of Time-Delay Systems . . . . . . . . . . . . . . 1

1.2.1 Smith-Predictor Control . . . . . . . . . . . . . 2

1.2.2 Finite Spectrum Assignment $\ldots \ldots \ldots \ldots$

1.3 Practical Issues . . . . . . . . . . . . . . . . 3

1.3.1 Automatic Tuning . . . . . . . . . . . . . . . 3

1.3.2 Continuous Self-Tuning . . . . . . . . . . . 4

1.3.3 Model Uncertainty and Controller Robustness . . . . . . 4

1.4 Real-Time Experimental Setup . . . . . . . . . . . 5

1.4.1 Process Simulator . . . . . . . . . . . . . 5

1.4 .2 Coupled-Tanks System . . . . . . . . . . . . . 5

2 FSA Algorithms Development 7

2.1 Basic FSA Algorithm . . . . . . . . . . . . . 7

2.2 Processes with Multiple Poles . . . . . . . . . . . . 11

2.3 Multivariable Processes . . . . . . . . . . . . 15

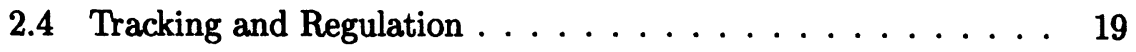

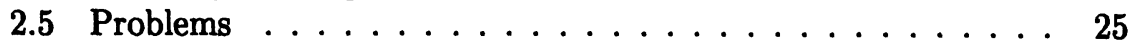

3 Closed-loop Process Identification 26

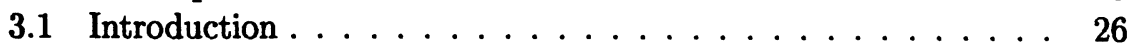

3.2 Basic Relay . . . . . . . . . . . . . . . . . 28

3.3 Relay with Bias . . . . . . . . . . . . . . . 30

3.3.1 Characteristics of Relay Oscillations . . . . . . . 30

3.3.2 Transfer Function Modelling . . . . . . . . . . 34

3.3.3 A Special Case . . . . . . . . . . . . . . . . 40

3.4 Relay Cascaded with a Nonlinear Mapping . . . . . . . . 42

3.4.1 Improved Estimation Accuracy . . . . . . . . . . . 42

3.4.2 Estimation of a General Point . . . . . . . . . . . . 49

3.4.3 Estimation of Multiple Points . . . . . . . . . . . . 52

3.5 Use of Relay Transients . . . . . . . . . . . . . . . . 54

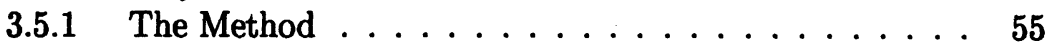

3.5.2 Simulation Study . . . . . . . . . . . . . . . . . 59

3.6 Frequency Response - Transfer Function Conversions . . . . . . 64

3.6.1 Single and Multiple Lag Processes . . . . . . . . . . 65

3.6.2 Second-order Modelling . . . . . . . . . . . . . 67 
viii

3.6.3 First-order Unstable Process with Time-delay . . . . . . 69

3.7 Problems . . . . . . . . . . . . . . . 71

4 Controller Design and Tuning $\quad 73$

4.1 Controller Design . . . . . . . . . . . . . . . . . . . 73

4.2 Auto-tuning . . . . . . . . . . . . . . . . . 74

4.3 Continuous Self-tuning . . . . . . . . . . . . . . 80

4.3.1 Process Estimation from Load Disturbance Response . 81

4.3.2 PID Adaptation . . . . . . . . . . . . 88

4.3.3 FSA Adaptation . . . . . . . . . . . . . . . 94

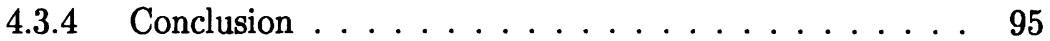

4.4 Unstable Processes . . . . . . . . . . . . . . . . . 95

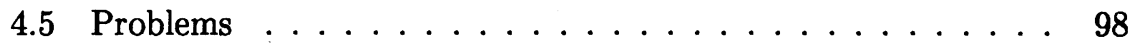

5 Robustness Analysis and Design 99

5.1 Practical Stability . . . . . . . . . . . . . . 99

5.2 Robust Stability . . . . . . . . . . . . . . . 105

5.3 Performance Robustness . . . . . . . . . . . . . . . 106

5.4 Robust Controller Design for First-Order Systems . . . . . . 108

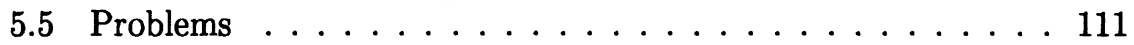

$\begin{array}{lr}\text { References } & 112\end{array}$ 